\title{
Geometry of Cores of Submodular Coherent Upper Probabilities and Possibility Measures
}

\author{
Tomáš Kroupa ${ }^{1}$
}

\begin{abstract}
We study and review geometrical properties of the set of the probabilities dominated by a submodular coherent upper probability (a possibility measure, in particular) on a finite set. We mention that there exists a polynomial algorithm for vertex enumeration. A new upper bound for the number of vertices in case of possibility measures is derived.
\end{abstract}

Key words: coherent upper probability, possibility measure, core

\section{Introduction}

In this contribution we study and review geometrical properties of the set of the probabilities dominated by a submodular coherent upper probability on a finite set. The aim is to identify those geometrical and algebraical properties that lead to the existence of efficient algorithms for processing the imprecise probability. One of the tasks of eminent importance is that of recovering the extreme points of the dominated set of probabilities or, at least, estimating their number. The class of imprecise probabilities for which both of these tasks are solvable are submodular coherent upper probabilities. We make an effort to single out some of its subclasses (such as possibility measures) to show that they exhibit additional properties from the geometrical viewpoint.

Basic concepts of imprecise probabilities [14] and possibility theory [4] are recalled in Sect. 2. The structure of cores is investigated in Sect. 3 and conclusions are in Sect. 4. In the paper we use definitions and results concerning polytopes (see [18], for example).

Institute of Information Theory and Automation of the ASCR, Pod Vodárenskou věží 4, 182 08 Prague, Czech Republic, kroupa@utia.cas.cz · Department of Mathematics, Faculty of Electrical Engineering, Czech Technical University in Prague, 16627 Prague 


\section{Basic Notions}

Let $N=\{1, \ldots, n\}$ be a finite set with $n \geq 2$ and let $2^{N}$ denotes the set of all subsets of $N$. A set function on $2^{N}$ is a mapping $\mu: 2^{N} \rightarrow \mathbb{R}$ with $\mu(\emptyset)=0$. We say that a set function is monotone when $\mu(A) \leq \mu(B)$ for every $A, B \in 2^{N}$ such that $A \subseteq B$; it is called submodular (or 2-alternating capacity) if the inequality $\mu(A \cup B)+\mu(A \cap B) \leq \mu(A)+\mu(B)$ holds for every $A, B \in 2^{N}$. When $P$ is a probability measure on $2^{N}$, then $p$ denotes the corresponding probability distribution on $N$, that is, the $n$-dimensional vector whose $i$-th component $p_{i}$ is $P(\{i\})$, for every $i \in N$. For any set function $\mu$ with $\mu(N)=1$, the set $\mathcal{M}(\mu)$ of probability distributions $p$ on $N$ with $P(A) \leq \mu(A)$, for every $A \subseteq N$, is called the core (or credal set) of $\mu$. Hence the core $\mathcal{M}(\mu)$ is the set of $n$-dimensional vectors $p \in \mathbb{R}^{n}$ satisfying the conditions

$$
\begin{aligned}
\sum_{i \in A} p_{i} & \leq \mu(A), \quad \text { for every } A \in 2^{N}, \\
p_{i} & \geq 0, \quad i=1, \ldots, n, \\
\sum_{i=1}^{n} p_{i} & =1 .
\end{aligned}
$$

Hence $\mathcal{M}(\mu)$ is a (possibly empty) convex polytope in $\mathbb{R}^{n}$ of dimension at most $n-1$. By ext $\mathcal{M}(\mu)$ we denote the set of all extreme points (vertices) of $\mathcal{M}(\mu)$, which is always finite because of the finite number of the affine constraints (1).

A coherent upper probability is a set function $\mu: 2^{N} \rightarrow[0,1]$ such that $\mu(A)=\sup \left\{\sum_{i \in A} p_{i} \mid p \in \mathcal{M}(\mu)\right\}$, for every $A \in 2^{N}$, and $\mathcal{M}(\mu) \neq \emptyset$. It can be deduced from a result of Walley [15, p. 14] that every submodular monotone set function $\mu$ with $\mu(N)=1$ is a coherent upper probability. On the contrary not every coherent upper probability is submodular - see [7, Remark 5.3]. A possibility measure is a set function $\Pi: 2^{N} \rightarrow[0,1]$ such that $\Pi(N)=1$, and where for every $A, B \in 2^{N}$, it holds that $\Pi(A \cup B)=$ $\max (\Pi(A), \Pi(B))$. A possibility distribution on $N$ is the $n$-dimensional vector $\pi$ whose $i$-th coordinate $\pi_{i}$ is $\Pi(\{i\})$, for every $i \in N$. Every possibility measure is submodular monotone and thus a coherent upper probability.

\section{Geometry of Cores}

The polytope $\mathcal{M}(\mu)$ is completely determined as the convex hull of the set of its vertices ext $\mathcal{M}(\mu)$. Vertex enumeration is the problem of generating all the vertices of a polytope given as an intersection of finitely many halfspaces. This is a fundamental problem in computational geometry, which is 
in general algorithmically highly nontrivial. Nevertheless there exist efficient techniques for certain classes of polytopes, which we mention in this section. It is therefore desirable to study the geometrical structure of the cores for particular classes of coherent upper probabilities in order to exploit their special properties. Since the time and the space complexity of the enumeration algorithms can also be judged a priori by estimating the number of vertices of the core, a goal in itself is to find some upper bounds for the number of vertices.

\subsection{Cores of Submodular Coherent Upper Probabilities}

The next theorem, which is considered to be well-known, gives a characterization of the core of submodular coherent upper probabilities. As far as the knowledge of the author goes, it can be traced back to Edmond's result [5] concerning the so-called base polyhedra in polymatroid ${ }^{1}$ theory [6]. Similar results appeared in coalition game theory and imprecise probabilities too, cf. [12] and [2]. The formulation below is based on the polymatroid version of the theorem from [6, Section 3.3].

Theorem 1. Let $\mu$ be a submodular coherent upper probability on $2^{N}$. Then $\mathcal{M}(\mu)$ coincides with the set of vectors $p \in \mathbb{R}^{n}$ satisfying the conditions (1a) and (1c). A vector $p \in \mathbb{R}^{n}$ is an element of $\operatorname{ext} \mathcal{M}(\mu)$ if and only if there exists an $(n+1)$-tuple of sets $A_{0}, \ldots, A_{n}$ belonging to $2^{N}$ such that

$$
\emptyset=A_{0} \subset A_{1} \subset \cdots \subset A_{n}=N
$$

where $A_{i} \backslash A_{i-1}=\left\{a_{i}\right\}$, for each $i=1, \ldots, n$, and

$$
p_{a_{i}}=\mu\left(A_{i}\right)-\mu\left(A_{i-1}\right), \quad \text { for each } i \in N \text {. }
$$

The complete description of the facial structure of $\mathcal{M}(\mu)$ can be found in [6, Theorem 3.30] (see also [13]). Note that the above theorem enables to dispense completely with the nonnegativity conditions (1b) defining the core $\mathcal{M}(\mu)$. This result justifies the game-theoretic terminology employed when calling the set $\mathcal{M}(\mu)$ "core" : for every submodular coherent upper probability $\mu$, the set $\mathcal{M}(\mu)$ coincides precisely with the core of the game $\mu$ as studied in coalition game theory. This fact however depends on the economic interpretation of the game $\mu$ since the game-theoretic core is usually defined with the reversed inequality in (1a) provided that the values of $\mu$ are profits resulting from the cooperation. The present inequality in (1a) thus implies that the value $\mu(A)$ should be thought of as a loss inflicted to a coalition $A$ rather

\footnotetext{
1 A polymatroid is a pair $\left(\mu, 2^{N}\right)$, where $\mu$ is a submodular monotone set function on $2^{N}$. When $\mu(N)=1$, then a so-called base polyhedron of the polymatroid $\left(\mu, 2^{N}\right)$ is precisely the core of $\mu$ in the sense of (1).
} 
than the profit generated by the coalition $A$. While the latter interpretation of coalition games is more common (cf. [12]), the first one also appears in the literature (for example, in the foundational Aubin's paper [1] about games with fuzzy coalitions).

Formula (2) also leads to a very inefficient algorithm for enumerating the vertices of $\mathcal{M}(\mu)$ that is based on generating all permutations of the elements of the set $N$. There exists, however, a vertex enumeration technique by Zhan [17], which is well-tailored to cores of submodular coherent upper probabilities. Zhan's algorithm is polynomial and enumerates all the vertices of $\mathcal{M}(\mu)$ in $O\left(n^{3}|\operatorname{ext} \mathcal{M}(\mu)|\right)$ time and in $O\left(n^{2}\right)$ space. We refrain from describing even the basic ideas of this sophisticated algorithm, which generalizes several enumeration methods. An interested reader is referred to [17] for the comprehensive details.

Submodularity is one of the properties enhancing the performance of the enumeration algorithms. Another property of a coherent upper probability leading to tractable computations is rather an intrinsic geometric property of its core: a $d$-dimensional polytope is called simple when each vertex is contained in precisely $d$ facets. The $d$-dimensional cube or simplex are examples of simple polytopes; the pyramid with a non-triangular base is not a simple polytope. Simplicity of the core enables us to recover the vertices efficiently since there exist enumerating algorithms running in polynomial time per vertex for the class of all simple polytopes (see [8] for details and references therein). The author of this paper proved recently in [8] that the core of every possibility measure is a simple polytope (see also Theorem 2 in this paper).

A very special core geometry arises from the example of Wallner in $[16$, p.347, Fig.2]: put $\mu(A)=1-f\left(\frac{n-|A|}{n}\right)$, where $f:[0,1] \rightarrow[0,1]$ is a strictly convex function with $f(0)=0, f(1)=1$. Namely, it is not difficult to prove that the core $\mathcal{M}(\mu)$ investigated in that example is combinatorially equivalent ${ }^{2}$ to the so-called permutahedron. Let $S_{n}$ be the set of all permutations of $N$. An $(n-1)$-dimensional permutahedron is the convex hull of the set $\left\{(\sigma(1), \ldots, \sigma(n)) \mid \sigma \in S_{n}\right\}$. The permutahedra are very rare among all polytopes: every $(n-1)$-dimensional permutahedron is a simple polytope that is an affine projection of the $\left(\begin{array}{c}n \\ 2\end{array}\right)$-dimensional cube [18, p.17].

It follows directly from Theorem 1 there are at most $n$ ! vertices in the core of every submodular coherent upper probability. More generally, the result of Wallner [16, Theorem 5.13] even shows that the submodularity condition can be relaxed so the upper bound is $n$ ! for every coherent upper probability. The next section is devoted to possibility measures for which some upper bounds for the number of vertices of their cores will be derived.

2 We say that two polytopes are combinatorially equivalent if there exists an orderpreserving bijection between their face lattices. 


\subsection{Cores of possibility measures}

When $\pi$ is a possibility distribution such that $\pi_{i}=0$ for every $i \in I$ with $I \subset N$, then, by the simple projection argument, the core of the possibility measure $\Pi$ can be identified with the core of the possibility measure $\Pi^{\prime}$ such that $\pi^{\prime}$ is defined as the restriction of $\pi$ to $N \backslash I$. Without loss of generality, we start with the following convention.

Convention. From now on we assume that $0<\pi_{1} \leq \cdots \leq \pi_{n}=1$.

Moral proved in [10] that $p \in \mathcal{M}(\Pi)$ if and only if $p$ is a probability distribution such that $\sum_{j=1}^{k} p_{j} \leq \pi_{k}$, for each $k=1, \ldots, n$. The cores of possibility measures were characterized in [8], where the proof of Theorem 2 can be found. Put

$$
S=\left\{i \in\{1, \ldots, n-2\} \mid \pi_{i+1}>\pi_{i}\right\} \cup\{n-1\} .
$$

Theorem 2. The core $\mathcal{M}(\Pi)$ of a possibility measure $\Pi$ is a simple $(n-1)$ dimensional polytope such that $p \in \mathcal{M}(\Pi)$ if and only if

$$
\begin{aligned}
\sum_{j=1}^{k} p_{j} & \leq \pi_{k}, \quad k \in S \\
p_{i} & \geq 0, \quad i=1, \ldots, n-1 \\
p_{n} & =1-\sum_{i=1}^{n-1} p_{i} .
\end{aligned}
$$

Moreover, the polytope $\mathcal{M}(\Pi)$ has $n-1+|S|$ facets given by $\mathcal{K} \cap \mathcal{M}(\Pi)$, where $\mathcal{K}$ is either $\left\{p \in \mathbb{R}^{n} \mid p_{i}=0\right\}$ or $\left\{p \in \mathbb{R}^{n} \mid \sum_{j=1}^{k} p_{j}=\pi_{k}\right\}$, for each $i=1, \ldots, n-1$ and for each $k \in S$, respectively.

The representation of $\mathcal{M}(\Pi)$ by the system (3) is irreducible, that is, removing any inequality or equation from (3) changes the set $\mathcal{M}(\Pi)$. While the irreducible representation (3) is easily constructed ${ }^{3}$ for every possibility measure, it can be shown that this is not the only irreducible representation of $\mathcal{M}(\Pi)$. The representation by system (3) is however useful for obtaining some upper bounds for the number of vertices. Miranda et al. [9] derived the exponential upper bound $2^{n-1}$ for the number $|\operatorname{ext} \mathcal{M}(\Pi)|$ of vertices of any possibility measure $\Pi$. Using only the simplicity of $\mathcal{M}(\Pi)$, the following lower bound and the upper bound for $|\operatorname{ext} \mathcal{M}(\Pi)|$ were obtained in $[8]^{4}$ :

$$
|S|(n-2)+2 \leq|\operatorname{ext} \mathcal{M}(\Pi)| \leq\left(\begin{array}{c}
n-2+|S|-r_{1} \\
r_{2}
\end{array}\right)+\left(\begin{array}{c}
n-2+|S|-r_{2} \\
r_{1}
\end{array}\right),
$$

\footnotetext{
${ }^{3}$ Detecting whether an inequality is redundant in a description of a polytope is a nontrivial problem, cf. [18, p. 48].

${ }^{4}$ Unfortunately, the formula for the upper bound is misprinted in [8, Theorem 2].
} 
where $r_{1}$ is the greatest integer such that $r_{1} \leq \frac{n-2}{2}$, and $r_{2}$ is the greatest integer such that $r_{2} \leq \frac{n-1}{2}$. It was shown in [8] that the upper bound from (4) is not uniformly better than the exponential bound. In the sequel we will show that there exists an easily computable upper bound that is always lower than the exponential bound. To this end, put $i_{0}=0$ and let $i_{1}, \ldots, i_{|S|}$ denote the elements of $S$ such that $i_{j}<i_{j+1}$, for each $j=1, \ldots,|S|-1$.

Theorem 3. If $\mathcal{M}(\Pi)$ is the core of a possibility measure $\Pi$, then

$$
|\operatorname{ext} \mathcal{M}(\Pi)| \leq 2^{|S|} \prod_{j=1}^{|S|}\left(i_{j}-i_{j-1}\right) .
$$

The expression on the right-hand side of (5) is always smaller or equal to $2^{n-1}$ with the equality holding when $\Pi$ is such that $\pi_{i}<\pi_{i+1}$, for each $i=1, \ldots, n-2$.

Proof. It follows from Theorem 2 and the definition of a vertex that there is a one-to-one correspondence between the vertices of $\mathcal{M}(\Pi)$ and uniquely solvable systems of $n-1$ linear equations selected from

$$
\begin{aligned}
\sum_{j=1}^{k} p_{j} & =\pi_{k}, \quad k \in S, \\
p_{i} & =0, \quad i=1, \ldots, n-1 .
\end{aligned}
$$

Hence it suffices to bound from above the total number of such uniquely solvable systems. Note that (6a) can be equivalently written as

$$
\begin{aligned}
& p_{1}+\ldots+p_{i_{1}} \quad=\pi_{i_{1}} \\
& p_{i_{1}+1}+\ldots+p_{i_{2}} \quad=\pi_{i_{2}}-\pi_{i_{1}} \\
& \ddots \quad(7) \\
& p_{i_{|S|-1}+1}+\ldots+p_{i_{|S|}}=\pi_{i_{|S|}}-\pi_{i_{|S|-1}}
\end{aligned}
$$

A uniquely solvable linear system arising from (7) by appending arbitrarily chosen equations from (6b) is called an initial linear system. First, we will count the total number of initial linear systems. Since the right-hand sides of (7) are positive due to our Convention, it follows that precisely one variable in each linear equation of (7) must be nonzero. This means that there are

$$
i_{1}\left(i_{2}-i_{1}\right) \ldots\left(i_{|S|}-i_{|S|-1}\right)=\prod_{j=1}^{|S|}\left(i_{j}-i_{j-1}\right)
$$

initial linear systems.

To finish the proof of (5), observe that every uniquely solvable system of $n-1$ linear equations chosen from (6a)-(6b) corresponds to precisely one 
initial linear system in which some of the $|S|$ equations from (7) are possibly interchanged with those from (6b). Since there are precisely $2^{|S|}$ of all interchanges, there can be at most $2^{|S|}$ of the uniquely solvable systems resulting in this way from an initial linear system. Combining this with (8), the inequality (5) follows.

In order to prove the second assertion of the theorem, observe that $\sum_{j=1}^{|S|}\left(i_{j}-i_{j-1}\right)=i_{|S|}=n-1$. We obtain

$2^{|S|} \prod_{j=1}^{|S|}\left(i_{j}-i_{j-1}\right)=\prod_{j=1}^{|S|} 2\left(i_{j}-i_{j-1}\right) \leq \prod_{j=1}^{|S|} 2^{i_{j}-i_{j-1}}=2^{\sum_{j=1}^{|S|}\left(i_{j}-i_{j-1}\right)}=2^{n-1}$

since $2 p \leq 2^{p}$ for every positive integer $p$.

In general, the upper bound from Theorem 3 is not better than the upper bound from (4) and vice versa. Note that the first part of the proof of Theorem 3 is based on a particular vertex enumeration algorithm, which can be used to recover the vertices when $n$ and $|S|$ are rather small. However, this technique is of a very limited use as it recovers the vertices in time exponential in $|S|$.

\section{Conclusions}

In the paper we presented the algebraical and the geometrical properties of imprecise probabilities that facilitate computations with their cores. Namely, there exist polynomial algorithms for vertex enumeration of both submodular coherent upper probabilities and coherent upper probabilities whose core is a simple polytope. The upper bounds from Sect.3.2 provide the preliminary judgment on the performance of such algorithms. There are many theoretical and practical facets of imprecise probabilities in which the computational properties of the core play a crucial role. For example, a concept of (conditional) independence can be defined with the help of extreme points of the core $[3,11]$.

An interesting open question is to describe the class of coherent upper probabilities whose cores are simple polytopes.

Acknowledgements The author owes his gratitude to his colleague Fero Matúš who attracted his attention to the connection between the cores of imprecise probabilities and the base polyhedra in polymatroid theory. The work of the author was supported by the grant No.1M0572 of the Ministry of Education, Youth and Sports of the Czech Republic. 


\section{References}

[1] Aubin JP (1974) Coeur et valeur des jeux flous à paiements latéraux. C R Acad Sci Paris Sér A 279:891-894

[2] Chateauneuf A, Jaffray JY (1989) Some characterizations of lower probabilities and other monotone capacities through the use of Möbius inversion. Math Social Sci 17(3):263-283

[3] Couso I, Moral S, Walley P (1999) Examples of independence for imprecise probabilities. In: Proceedings of the 1st International Symposium on Imprecise Probabilities and Their Applications ISIPTA, pp 121-130

[4] Dubois D, Prade H (1988) Possibility theory. Plenum Press, New York

[5] Edmonds J (1970) Submodular functions, matroids, and certain polyhedra. In: Combinatorial Structures and their Applications (Proc. Calgary Internat. Conf., Calgary, Alta., 1969), Gordon and Breach, New York, pp 69-87

[6] Fujishige S (1991) Submodular functions and optimization, Annals of Discrete Mathematics, vol 47. North-Holland Publishing Co., Amsterdam

[7] Krätschmer V (2003) Coherent lower previsions and Choquet integrals. Fuzzy Sets and Systems 138(3):469-484

[8] Kroupa T (2008) Geometry of possibility measures on finite sets. Internat J Approx Reason 48(1):237-245

[9] Miranda E, Couso I, Gil P (2003) Extreme points of credal sets generated by 2-alternating capacities. Internat J Approx Reason 33(1):95-115

[10] Moral S (1985) Información difusa. relaciones entre probabilidad y posibilidad. PhD thesis, Universidad de Granada

[11] Moral S, Cano A (2002) Strong conditional independence for credal sets. Ann Math Artif Intell 35(1-4):295-321

[12] Shapley LS (1972) Cores of convex games. Internat J Game Theory $1: 11-26$

[13] Verdegay-López JF, Moral S (2000) Network of probabilities associated with a capacity of order-2. Inform Sci 125(1-4):187-206

[14] Walley P (1991) Statistical reasoning with imprecise probabilities, Monographs on Statistics and Applied Probability, vol 42. Chapman and Hall Ltd., London

[15] Walley P (1996) Measures of uncertainty in expert systems. Artificial Intelligence 83(1):1-58

[16] Wallner A (2007) Extreme points of coherent probabilities in finite spaces. Internat J Approx Reason 44(3):339-357

[17] Zhan P (1997) A polynomial algorithm for enumerating all vertices of a base polyhedron. J Oper Res Soc Japan 40(3):329-340

[18] Ziegler G (1995) Lectures on polytopes, Graduate Texts in Mathematics, vol 152. Springer-Verlag, New York 